\title{
Getting a brighter view on animal anatomy
}

\author{
Ellen P. Neff
}

F rank van Breukelen can place a port into the jugular vein of a rat in a matter of minutes. The first time he had to perform the procedure in a tenrec, it took him two hours. Tenrecs are, in van Breukelen's words, a really weird group of animal. That makes them exciting to work with, but a challenge at the same time. $\mathrm{He}$ has spent the past five years getting a colony of common tenrecs, Tenrec ecaudatus, established in his lab at the University of Nevada, Las Vegas (UNLV). He's become more adept at working with them along the way but still, just not much is known about these odd little creatures.

The tenrecs are indeed unusual among mammals and are thought to be one of the most basal placental animals out there. The tenrecs-a family of 35 species native to Madagascar and parts of Africa-are diverse in size and appearance as well as the myriad habitats and ways in which they live. They're mammalian in some ways but almost reptilian in others. van Breukelen went through the trouble of bringing T. ecaudatus into his lab to study how they hibernate, which differs quite from the more widely studied ground squirrel. Studying 'oddities' in the animal kingdom is part of solving the puzzles of life, but access to the more unusual animals can be an issue. Others are interested in the tenrec, van Breukelen says, but the animals are hard to come by. Instead of physical specimens, he's hoping there will soon be a digitally dissectible version to distribute.

When UNLV established its medical school in 2017, a common element of medical pedagogy was conspicuous in its absence: cadavers. Instead, medical students tackle their gross anatomy huddled around one of several Sectra tables, upon which they complete virtual dissections with a dissector platform from Aurora, Coloradobased Touch of Life Technologies that's built with images from the National Library of Medicine's Visible Human Project. The company is focused on providing educational tools for human anatomy, but animals are a possibility too. Including van Breukelen's tenrecs.

While working with Touch of Life to develop a human anatomy course a few years ago, van Breukelen brought up his unusual animals. A tenrec that had died soon found

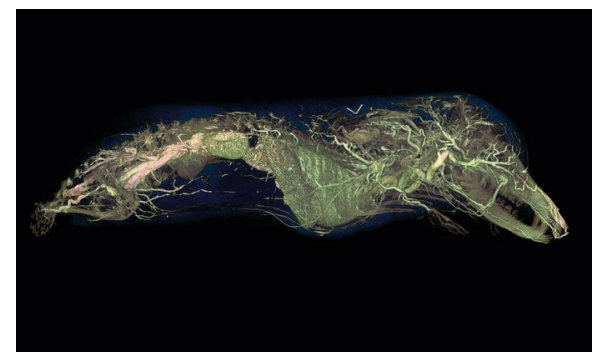

Bright inside: A CT scan of BriteVu-perfused tenrec from van Breukelen's lab. Credit: Scott Echols, Scarlet Imaging

itself in Colorado, where Touch of Life meticulously sectioned the $\sim 25$ centimeter animal into about 5000 50-micron slices for scanning and reassembly into a digital version. The first iteration, compared against a CT scan of the tenrec, gave van Breukelen a unique view into his unique animal, but there was a slight hitch: with no real reference to start with, it was difficult to register all those slides together. "If you're slightly off in the photos, it doesn't work all that well," says van Breukelen.

In need of better contrast to assist alignments, Touch of Life brought in Scott Echols, an avian veterinarian who, at home in his kitchen, had developed a novel terminal contrast agent called BriteVu that is perfused through a euthanized animals' vascular system to allow detailed imaging, via CT or X-ray scans, of blood vessels from large arteries to miniscule capillaries. The work started with African gray parrots but since he developed the agent in 2014, Echols has perfused and imaged animals from zebrafish and rodents to horses and even humans.

The BriteVu-perfused tenrec is still in assembly at Touch of Life, but van Breukelen and Echols hope it is just the beginning. Though tactile responses may be absent, having a three-dimensional representation of an animal can expand access to that animal itself and help researchers and veterinarians gain better perspectives about its anatomy, says Echols. "For someone like myself, I'm a bird surgeon-I use this information to guide surgeries." For him, it all goes back to understanding what is 'normal' for a given animal (and he would like to see detailed images of every animal he

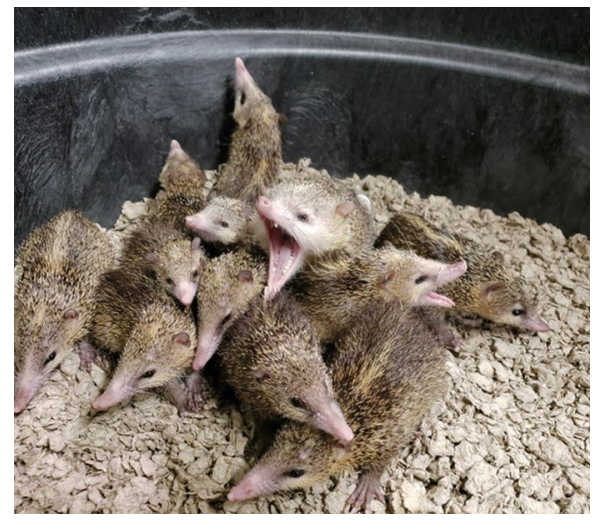

In the fur: tenrecs in van Breukelen's lab. Credit: Gilbecca Smith

can find to collaborate on). To understand abnormal-that is, a disease-you have to be able to recognize healthy anatomy too, he says But when an animal is physically dissected, that animal is destroyed; advanced digital imaging methods, completed before traditional histopathology, can preserve it for others to study for years to come, he says.

That might be somewhat less of a concern for commonly used research species where specimens are abundant, but those represent a small fraction of diversity out there. "There are other researchers out there who have really bizarre animals that nobody has access to," says van Breukelen. "I'm really intrigued about the idea of creating a dissectible zoo, if you will, for the Sectra Table." There are benefits to teaching and comparative anatomy research, he saysthe digital version can be easily annotated and shared, without requiring the sacrifice of additional animals - and for practical in vivo work as well. A better anatomical map can save some of the learning curve with working with an unfamiliar animal as well. "Having an idea of the general layout of an organism would make your life so much easier," van Breukelen says.

Ellen P. Neff

Lab Animal.

e-mail:ellen.neff@us.nature.com

Published online: 8 July 2019

https://doi.org/10.1038/s41684-019-0355-1 